\title{
Archéopages
}

Archéopages

Archéologie et société

40 | 04-07/2014

Villages

\section{Chronologie de l'occupation de la villa de la Lesse à Sauvian basée sur l'étude des céramiques issues des puits}

Pierre Rascalou

\section{CpenEdition}

Journals

Édition électronique

URL : https://journals.openedition.org/archeopages/655

DOI : 10.4000/archeopages.655

ISSN : 2269-9872

Éditeur

INRAP - Institut national de recherches archéologiques préventives

Édition imprimée

Date de publication : 1 juin 2015

Pagination : 166-168

ISSN : 1622-8545

\section{Référence électronique}

Pierre Rascalou, «Chronologie de l'occupation de la villa de la Lesse à Sauvian basée sur l'étude des céramiques issues des puits ", Archéopages [En ligne], 40 | 04-07/2014, mis en ligne le 01 juillet 2016, consulté le 26 janvier 2022. URL : http://journals.openedition.org/archeopages/655 ; DOI : https:// doi.org/10.4000/archeopages.655 


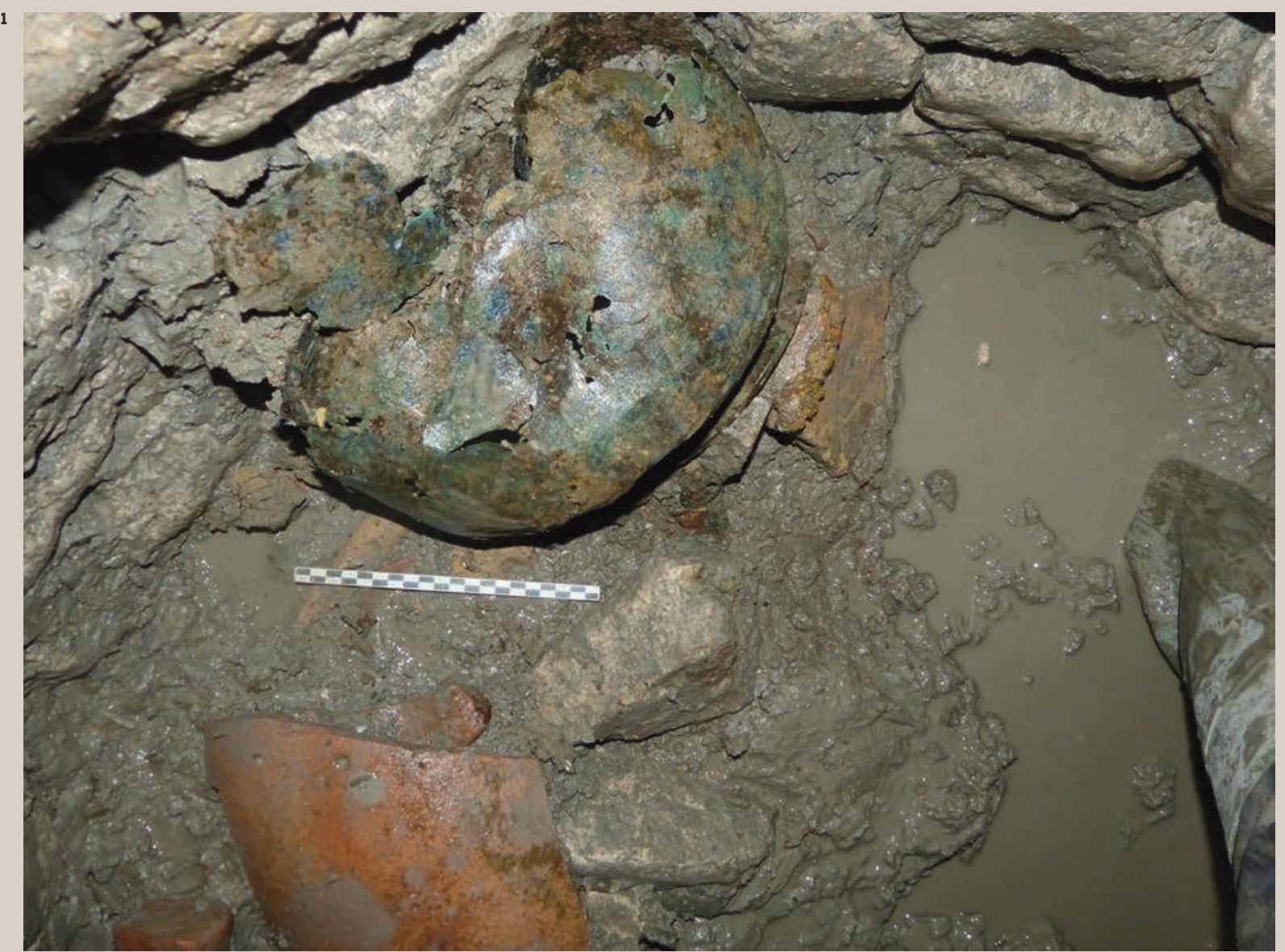

un long portique adossé à la limite septentrionale de la grande esplanade.

Deux des cinq puits mis au jour sur le site ont fait l'objet d'une étude intégrale, les moyens alloués à l'opération ne permettant pas d'envisager de fouiller l'ensemble des structures. Les constructions choisies l'ont été en raison de leur attribution chronologique: chacune correspondant à l'une des deux phases d'occupation déduite de l'étude stratigraphique. Il était par conséquent possible d'escompter la découverte d'indices en mesure d'argumenter non seulement le mode de fonctionnement du temple voisin (dédicaces, offrandes...), mais également les éventuelles spécificités du vaisselier réservé au service des pèlerins - en sus, bien sûr, des informations liées à leur alimentation et des éclairages paléoenvironnementaux attendus de la découverte de graines ou de restes de fruits. Le puits le plus ancien s'est avéré décevant. Profond de $7 \mathrm{~m}$ et dépourvu d'enveloppe construite, il n'a livré que très peu de renseignements. Seule la partie sommitale du remplissage incluait deux amphores italiques, ainsi que plusieurs centaines de tessons provenant de contenants vinaires du même type.

La seconde construction, en revanche, a révélé la présence d'une stratigraphie dont l'ensemble des recharges superposées sur une épaisseur de $17 \mathrm{~m}$ (couches de fonctionnement et remblais d'abandon intermédiaires) abritait un mobilier très abondant ainsi qu'une grande quantité d'objets en bois et de macro restes (fruits essentiellement: noix, noisettes, raisins...). Aucun élément en rapport direct avec le sanctuaire n'a été mis au jour. La céramique est foisonnante. Une quinzaine de récipients complets a été remontée en surface (dont trois en bronze) [ill. 1], ainsi que plusieurs milliers de tessons dont l'étude (à venir) permettra de faire la part entre les matériels de puisage (des cruches le plus souvent) et les vases de stockage qui permettaient d'immerger certains aliments, ainsi conservés quelque temps dans la fraîcheur du réservoir aménagé dans la partie inférieure du conduit.

\footnotetext{
Références bibliographiques

Ginouvez O., 2013: Hérault, Magalas, Le Pendut. Projet de lotissement Les terrasses de Montfau, Rapport final d'opération, diagnostic archéologique, Inrap Méditerranée.
}

\section{Chronologie de l'occupation de la villa de la Lesse à Sauvian basée sur l'étude des céramiques issues des puits \\ Pierre Rascalou, Inrap, UMR 5140 «Archéologie des sociétés méditerranéennes»}

La fouille de puits apporte généralement un volume considérable de mobiliers. Pour le céramologue, cela signifie la gestion de nombreuses caisses encombrantes et l'étude de milliers de tessons appartenant à quelques 
$0 \mathrm{~m}-\quad 0 \mathrm{~m}-$

$1 \mathrm{~m}-$
$2 \mathrm{~m}-$
$3 \mathrm{~m}-$
$4 \mathrm{~m}-$
$5 \mathrm{~m}-$
$4 \mathrm{~m}-$
$5 \mathrm{~m}-$

000

600

200

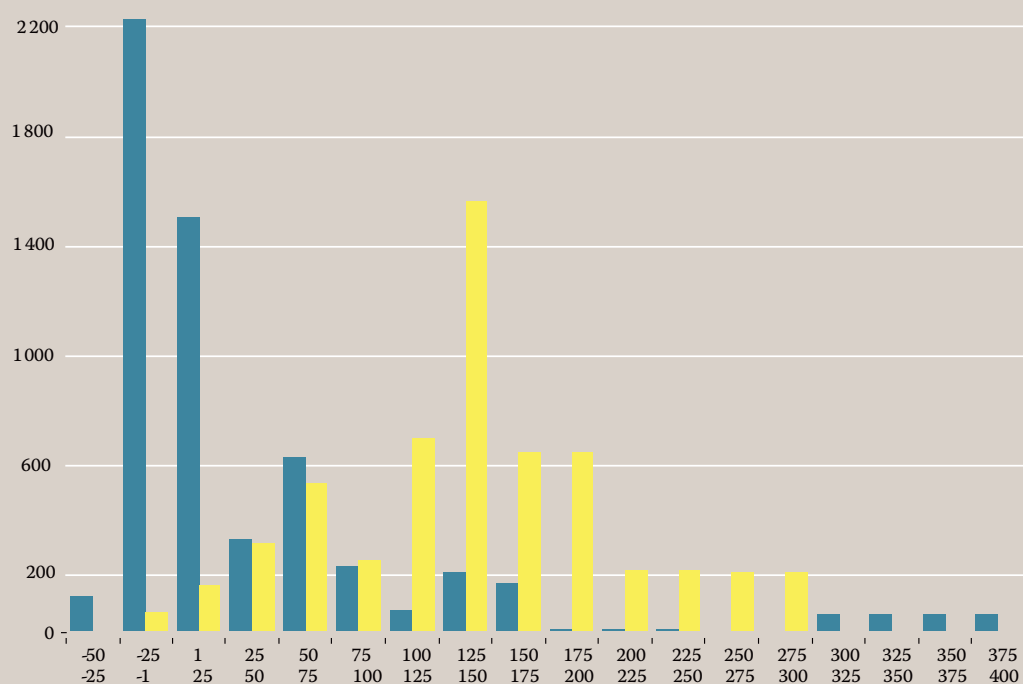

Nfr de céramiques hors puits

(rapport entre la part des comblements et la proportion des céramiques sur l'ensemble du remplissage du puits)
Absence ou faible $(<0,25)$

Moindre (0,25-0,75)

Conforme $(0,75<1,5)$

Forte $(>1,5,<2,5)$

Surreprésenté $(>2,5)$

$\mathrm{Nfr}$ de céramiques des puits

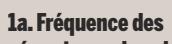

céramiques dans les puits

de la Lesse.

1b. Répartition par quart de siècle du nombre de céramiques découvertes sur la villa de la Lesse. dizaines de vases, essentiellement des cruches qu'il faut au préalable tenter de reconstituer. Le remontage des vases prend beaucoup de temps sur celui attribué à l'étude des céramiques. C'est pourtant une étape obligée. Il suit un protocole d'inventaire prenant en compte les caractères technologiques et typologiques, la fragmentation et l'état de conservation du vase, la présence et l'aspect de la poix encore adhérente aux parois des tessons. Ce remontage permet d'envisager des présentations muséographiques, d'apporter des précisions typochronologiques pour des formes connues jusque-là uniquement par des fragments ou encore de calculer la contenance de vases complets. Il est encore nécessaire afin de mieux isoler les vases en situation fonctionnelle de ceux présents de façon plus fortuite ou résiduelle. Il permet ainsi une bien meilleure approche chronologique sur des lots particulièrement difficiles à dater, car essentiellement composés de céramiques communes. Les fréquences des céramiques ou de certaines catégories, établies de façon la moins subjective possible, aident à caractériser les types de comblement, qu'ils soient liés à l'utilisation, la désaffection ou la condamnation du puits [ill. 1a]. Visuellement, les niveaux de fonctionnement sont mieux perçus. Les remblais ou les remplissages naturels, avec de plus faibles concentrations ou des mobiliers hétérogènes, occupent en général les parties supérieures. Dans le détail, les variations de fréquences peuvent aussi suggérer l'utilisation préférentielle de seaux ou l'usage de conteneurs en matériaux périssables. L'examen des céramiques issues des puits ne se réduit pas aux seuls champs de la céramologie. Il doit contribuer à une meilleure perception du statut du site, de son économie et de son évolution. Les décapages réalisés sur la villa de la Lesse à Sauvian (Hérault) ont mis au jour deux puits (Pomarèdes, 2011). Un premier est situé en bordure d'une cour centrale, tandis que le second est positionné plus à l'écart des bâtiments.

Une grande partie des céramiques collectées au cours de la fouille provient d'ensembles datés autour du changement d'ère. À partir de l'époque flavienne, les témoins se font beaucoup plus discrets. Mis à part quelques niveaux tardifs en lien avec la récupération de matériaux, la plupart des niveaux d'occupation paraissent antérieurs au milieu du $\mathrm{II}^{\mathrm{e}}$ siècle de notre ère. De façon assez logique, cette situation pourrait s'expliquer par un abandon rapide du site. La mauvaise conservation de niveaux supérieurs sera avancée, notamment au regard de mobiliers plus récents parfois rencontrés hors stratigraphie. On nuancera un cadre chronologique réduit, souvent le résultat de la fouille de vestiges marquant les phases de construction ou de réaménagement. C'est sans doute le cas pour la villa de la Lesse. Une fois atteint son optimum de 
développement, le site ne fournit plus, ou peut-être pas assez pour être perceptibles, de rejets permettant de prolonger son occupation au $\mathrm{II}^{\mathrm{e}}$ siècle, voire même au $\mathrm{III}^{\mathrm{e}}$ siècle de notre ère. Pour illustrer ces périodes, on ne dispose plus de structures en creux, ces pièges à artefacts que sont les fosses d'extraction de matériaux ou les tranchées d'implantation de constructions.

L'examen des céramiques issues des puits présente une image bien différente sur la chronologie de l'occupation. On y rencontre surtout du matériel en usage au $\mathrm{II}^{\mathrm{e}}$ siècle et encore au $\mathrm{III}^{\mathrm{e}}$ siècle de notre ère [ill. 1b]. Une opération archéologique menée sans la fouille des puits nous aurait probablement conduits à envisager un abandon précoce du site au profit de la villa de la Domergue. Ce grand domaine est établi à moins de $1 \mathrm{~km}$ au nord et son occupation se prolonge jusquà la fin de l'Antiquité. La situation paraît plus complexe si l'on envisage comme possible le maintien d'une occupation et d'activités plus discrètes sur le site.

La fouille des puits de la Lesse permet d'illustrer les problèmes de taphonomie que l'on peut rencontrer à la surface des sites et d'apporter des nuances sur les modèles d'implantation et de disparition des habitats ruraux antiques.

\footnotetext{
Références bibliographiques

POMARÈDES H. (DIR.), 2011, Implantation d'une ferme pré-augustéenne et développement d'une petite villa de la cité de Béziers (fin $I^{e r}$ s. av. /III $s$. ap. J.-C.), La Lesse, Sauvian (Hérault), Rapport final d'opération, Inrap Méditerranée, 377 p.
}

Des qanàts en Gaule Narbonnaise

Roland Haurillon, Inrap

Avec l'intervention de l'association Archéopuits, une équipe de l'Inrap a pu étudier trois puits antiques au nord-est de Béziers (Hérault) (Haurillon et al., 2011 et 2012 ; Haurillon, 2012). Situés en contexte rural, hors habitat, carrés ou circulaires, parfois bâtis, ils ont une profondeur de 5,10 et $11,5 \mathrm{~m}$. Grâce à leur fouille exhaustive, nous avons pu mettre en évidence leur relation avec des galeries drainantes souterraines appartenant à trois réseaux différents.

Le milieu hydrogéologique local est propice à la formation de petits aquifères. Pour récupérer cette précieuse ressource, les Romains utilisent un système d'exploitation en puits-galerie. Le procédé consiste à creuser à la sape un tunnel quasi horizontal à partir de puits verticaux alignés et équidistants. Les nappes perchées sont alors traversées par certains de ces puits (puits-mères) dont l'eau est récupérée par ladite galerie. La récolte se fait donc ici par captage, mais également par percolation [ill. 1]. En effet, la galerie souterraine draine aussi l'eau contenue dans l'aquifère gréseux dans laquelle elle a été creusée. Cette eau exsude sous forme de fines gouttelettes le long des parois. C'est la récolte des «larmes» ou «pleurs de terre», expression utilisée dans la littérature archéologique du XVIII ${ }^{\mathrm{e}}$ siècle pour décrire ce phénomène déjà observé dans d'autres ouvrages mal datés, au nord de Béziers (Sabatier, 1854). L'eau est ensuite acheminée par une rigole aménagée à la base de la galerie souterraine, jusquà une vaste citerne enterrée. Un tel stockage d'appoint permet ainsi d'alimenter certaines activités domestiques, agricoles ou artisanales.

Ce système hydraulique reconnu sur le territoire de Béziers est analogue à des ouvrages recensés sur tout le pourtour méditerranéen (Goblot, 1979). Ils portent le nom de « qanàt». Si le milieu scientifique s'accorde à lui donner une origine eurasienne qui remonterait au $\mathrm{I}^{\mathrm{er}}$ millénaire avant notre ère, ce savoir-faire serait encore plus ancien puisqu'il hériterait de techniques minières. Quelques ouvrages similaires ont été étudiés, principalement en Gaule Belgique (Kayser, Waringo, 2003; Boulanger, 2009). Cependant, c'est la première fois que la fouille d'une série de puits met en évidence l'utilisation de cette technique en Gaule Narbonnaise. Associées à celles présentées dans cette rubrique, ces découvertes biterroises confirment l'intérêt d'une collaboration avec Archéopuits, dans le cadre de ce type d'intervention archéologique. Elle doit bien évidemment être stimulée par une volonté scientifique pour faire aboutir une réflexion ouverte sur la problématique de ces ouvrages. 